\title{
HOT CARRIER EFFECTS IN OPTICALLY DETECTED CYCLOTRON RESONANCE STUDIES OF III-V SEMICONDUCTORS*
}

\author{
K. KarpińsKa, S. DedulewiCZ ${ }^{\dagger}$ and M. GodlewsKi \\ Institute of Physics, Polish Academy of Sciences \\ Al. Lotników 32/46, 02-668 Warsaw, Poland
}

\begin{abstract}
The mechanisms of irregular photoluminescence intensity oscillations, as observed in optically detected cyclotron resonance experiments, are discussed. Two possible scenarios are analyzed, both requiring impact ionization of the center(s) by electric field accelerated free carriers. The first assumes coexistence of dielectric and energy relaxation processes. The second assumes a subsequent impact ionization of two different centers.

PACS numbers: 71.35.+z, 72.70.+m, 76.90.+d
\end{abstract}

\section{Introduction}

It was demonstrated in an earlier work that cyclotron resonance (CR) can be detected optically via characteristic changes of the photoluminescence (PL) intensity [1]. In the case of "edge" emissions in pure materials optically detected cyclotron resonance (ODCR) may be detected due to the shift of emission spectra towards higher energies following an increase in free carricrs energy. The mechanism of ODCR detection for doped semiconductors was explained in Ref. [2]. It was proven experimentally that impact ionization processes can result in a relatively large changes of PL intensity and in large ODCR signals [2]. This is explained as follows: Microwave heated free carriers at CR condition can gain sufficient energy for the impact ionization of shallow impurities and dissociation of bound excitons $[2]$.

It was also shown that the application of microwave power may cause irregular oscillations in free carrier density [3] and, as observed recently [4], of photoluminescence (PL) intensity. Possible mechanisms responsible for such oscillations are discussed in the present paper.

\section{Results and discussion}

In Fig. 1a we present the PL spectrum of $\mathrm{Ga}_{0.47} \operatorname{In}_{0.53} \mathrm{As}$ epilayer measured under $514.5 \mathrm{~nm}$ excitation at the temperature of $2 \mathrm{~K}$. The spectrum shown in Fig. 1b was taken under the same experimental conditions but with an external

*This work was partly supported by the grant no. 204769101 of the Committee for Scientific Research .

†Permanent address: Semiconductor Physics Institute, A. Goštauto 11, Vilnius, Lithuania. 
magnetic field set at the CR condition and with $200 \mathrm{~mW}$ of microwave power. It is observed that the whole PL spectrum oscillates. These oscillations have an irregular character, as can be observed in the inset of Fig. 1. This inset shows the temporal changes of the intensity of the donor-acceptor pair emission at $1572 \mathrm{~nm}$.

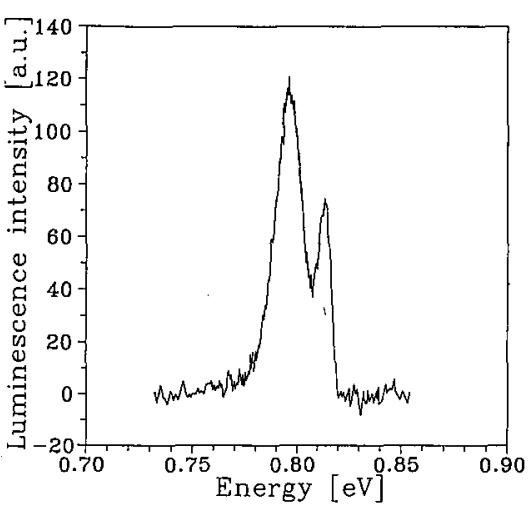

a)

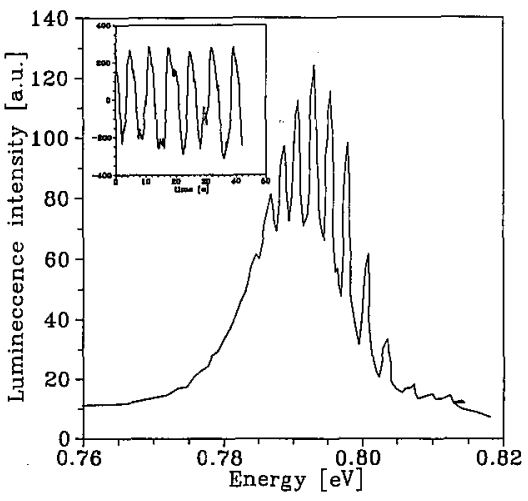

b)

Fig. 1. PL spectrum of the $\mathrm{Ga}_{0.47} \operatorname{In}_{0.53} \mathrm{As}$ crystal measured at $2 \mathrm{~K}$ and argon laser excitation. Spectrum shown in (b) was measured at the same conditions with magnetic field set at $\mathrm{CR}$ condition and $200 \mathrm{~mW}$ of the microwave power. The temporal changes in intensity of the $1572 \mathrm{~nm}$ emission are shown in the inset.

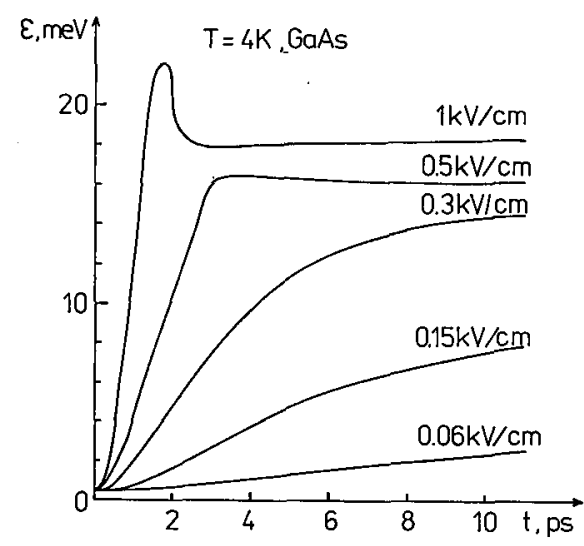

Fig. 2. Time deperldence of the mean electron energy for five different electric fields applied. The oscillation observed for $1 \mathrm{kVcm}^{-1}$ is due to optical phonon emission by an overheated electron. These oscillations are then quenched by carrier scattering on ionized impurities and on acoustic phonons.

Theoretical calculations were performed to estimate the mean energy which can be gained by electrons under the influence of relatively low electric fields. In 
Fig. 2 we show the time dependence of the mean electron energy for different electric fields applied. These data were obtained using typical material parameters of a III-V semiconductor in the calculations (GaAs parameters were taken). The details of the theoretical calculations can be found elsewhere [5]. These data prove that even for relatively low electric fields (in the range expected in the ODCR experiment) free carriers can accumulate sufficient energy for impact ionization of shallow centers and for exciton dissociation. This possibility was confirmed experimentally. The threshold dependence of the PL intensity on microwave power was observed. Such dependence is a fingerprint of the impact ionization mechanism. This confirmed the previous observation of one of the authors that the impact ionization process is responsible for the ODCR detection for doped materials [2].

We have reported recently the observation of a new phenomenon which accompanies the optical detection of CR [4]. In the case of GaInAs, it was observed that once the threshold power for impact ionization of the deeper of the two-donor centers present in the material is reached, a decrease of the relevant PL intensity is accompanied by an appearance of irregular oscillations of the intensity of the whole PL spectrum, as shown in Fig. 1. Such correlation between the appearance of oscillations and the achievement of the threshold for impact ionization is predicted by two theoretical models describing the scenarios for chaotic oscillations in free carrier density $[7,8]$. The first of them assumes a coexistence of dielectric relaxation and energy dissipation processes and impact ionization of one shallow-donor state [6]. The second model includes the subsequent impact ionization of two different states with the energy relaxation taken into account [7].

Both models should be analyzed in order to account for the experimental results obtained. GaInAs measurements show impact ionization of two different donor states [4]. Thus, the second model should be considered in this case. Our recent experiments performed for GaAs and AlGaAs [8] cannot be explained by this model. We have no evidence for subsequent impact ionization of two states in this case and, thus, the first model seems to be more appropriate.

Numerical calculations for both these models were performed. By selecting appropriate starting parameters we could reproduce the situation in which free carrier density begins to oscillate. We indicate, however, that the calculated oscillations have frequencies which are much higher than those observed in the experiment. Similar frequencies were obtained by previous theoretical calculations, even though the possibility that a low-frequency component appears was claimed to be a characteristic feature of impact ionization - related chaotic oscillations in materials with the low carrier mobility [7]. Low frequency of the oscillations that we observe is not understood by us at present. We indicate that this is an important problem to clear since low frequency oscillations were reported also in other cases [9] and are, for example, considered as a main source of false signals in telecommunication [10].

\section{References}

[1] R. Romestain, C. Weisbuch, Phys. Rev. Lett. 45, 2067 (1980).

[2] H. Weman, M. Godlewski, B. Monemar, Phys. Rev. B 38, 12525 (1988). 
[3] B.M. Ashkinadze, A.V. Subashiev, JETP Lett. 46, 358 (1987).

[4] M. Godlewski, K. Fronc, M. Gajewska, W.M. Chen, B. Monemar, Plıys. Rev. B 44, 8357 (1991).

[5] S. Dedulewicz, M. Godlewski, this conference.

[6] G. Hüpper, E. Schöll, L. Reggiani, Solid-State Electron. 32, 1787 (1989).

[7] E. Schöll, Appl. Phys. A 48, 95 (1989).

[8] M. Godlewski, unpublished.

[9] V.A. Samuilov, D.L. Pulfrey, Proc. MRS Spring Meeling, San Francisco 1992 (in press).

[10] M.L. Uren, Proc. MRS Spring Meeting, San Francisco 1992 (in press). 